\title{
The Power System Analysis Based on Neural Network CHEN Yang ${ }^{1, \text { a }}$
}

\author{
${ }^{1}$ Department of Electrical Information Engineering, Northeast Petroleum University, Daqing 163318, \\ China \\ a yongechan@163.com
}

Keywords: Deep Neural Network; Power System; System Performance; System Optimization.

\begin{abstract}
With the rapid and bursting development of pattern recognition technique and power system theory, the combination of the two is urgently needed. We argue that a filter bank that is designed from perceptual evidence is not always guaranteed to be the best filter bank in a statistical modeling framework where the end goal is word error rate. Therefore, to combine the proposed techniques with the neural network, we conduct theoretical analysis and numerical simulation on the power systems. The experimental analysis and numerical simulation indicate that our proposed approach performs well and contains satisfactory robustness.
\end{abstract}

\section{Introduction}

Data analysis procedures can be broadly categorized as either exploratory or confirmatory, based on the models used for processing the data source. Regardless the methods used in both categories, one key component is data grouping using either goodness-of-fit to a postulated model or clustering through analysis. Generator leading phase operation is a kind of economic and effective measures of voltage regulation and power quality improvement. Due to the synchronous generator is a multivariable and strong coupling nonlinear system it is difficult to obtain satisfactory results by traditional analysis method [1-3]. This paper proposes a new method of modeling generator leading phase ability based on radial basis function (RBF) neural network. Because of generator leading phase operation test can't be poor for all point, therefore, how to use a typical test results into a phase ability to infer the actual running conditions of generator in phase and the generalization of generator in phase test outcomes problem worth studying.

Generators in phase ability of traditional modeling method basically has the following kinds: (1) Assume that the synchronous reactance unsaturated mathematical model of generator in phase each value; (2) According to the assumption of constant voltage of the machine of generator in phase mathematical model between each quantity; (3) Hypothesis test results of each quantity of linear fitting into the phase of results curve model. These models are widely used in engineering practice. The synchronous generator leading phase operation parameters of the characteristics of nonlinearity, randomness and interference and network parameters is easy to change [4]. Therefore, the established on the basis of experiment and hypothesis model is very difficult to exactly reflect the change of generator in phase parameters and the applicable scope is limited. Chaos phenomenon widely exists in natural system and social system which is one between sure and random irregular movement is a kind of complex behavior generated by the nonlinear dynamic system. The essential characteristic of chaotic systems is the system extreme sensitivity to initial conditions. Chaos in many practical systems have become more widely used such as biomedical, electric power, transportation, communication, social economy, acoustics, chemistry and so on. Despite the chaos phenomenon is an irregular phenomenon, it is the result of a deterministic system. As the result, in the short range is predictable. Combining the chaos phenomenon with neural network is becoming the research hot topic in the computer science community. Convolutional neural networks (CNNs) are a specific type of DNN that have shown state-of-the-art performance across a variety of small and large vocabulary tasks. The most popular features to use with CNNs are hand-crafted filter bank features. The multi-filter bank is inspired by auditory and physiological evidence of how humans perceive speech signals. We argue that a filter bank that is designed from perceptual evidence is not always 
guaranteed to be the best filter bank in a statistical modeling framework where the end goal is word error rate. Therefore, to combine the proposed techniques with the neural network, we conduct theoretical analysis and numerical simulation on the power systems which are shown in the following sections.

\section{The Concepts of the Proposed Approach}

The Principles of Neural Network. Neural network is imitation and extension of the human brain cognitive function of new type of intelligent information processing system, it is made of a large number of simple processing elements (neurons) of a complex nonlinear adaptive dynamic learning system. Artificial neural network technology as a new means of artificial intelligence technology is currently one of the research direction of rapid development in the world [4-5]. Artificial neural network with a large number of neurons widely connected to each other and constitute a complex network system, to some extent, it mimics the cranial nerve system of information processing, storage and retrieval functions, learning, memory and computing intelligence processing capacity. Artificial neural network in the optimization, signal processing and pattern recognition, intelligent control, fault diagnosis and many other fields has a broad application prospect.

BP neural network is a multilayer of reverse transmission and the ability to correct error mapping function. It through to the unknown system input and output parameters after learning which can express the associative memory system. The learning process of BP neural network by signal is spread and error back propagation of two parts. Forward propagation denoted as the incoming sample input from the input layer is bamboozled by hidden layer to output layer [6-7]. If output layer does not accord with the desired output, the output error back propagation step by step through the hidden layer to the input layer which will influence the error spread to all the nodes in each layer serves as the basis of the correct weights of each node is connected which is shown below figure 1 .

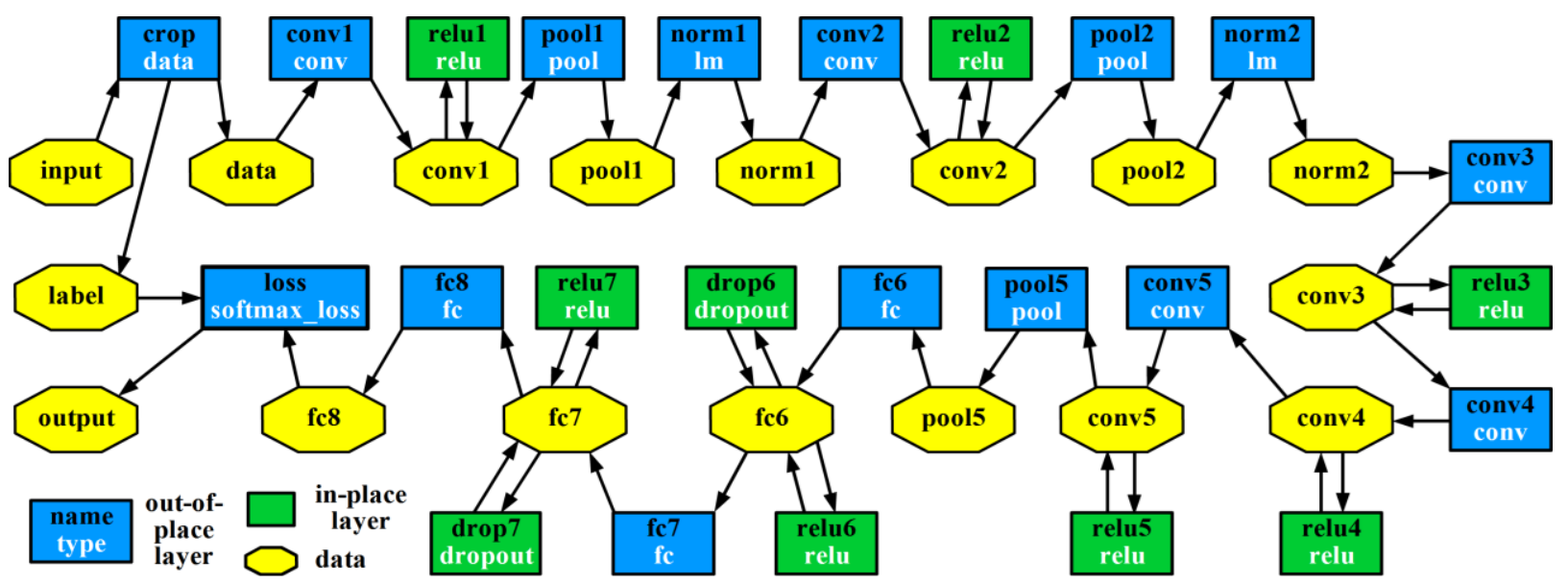

Fig. 1The General Structure of the Proposed Neural Network

Note that the input into the filter learning stage is the normalized log of the power spectrum, which is then taken to be positive using the exponent. The following formula $1 \sim 3$ shows the process. The equation 1 shows the input of each node in hidden layer. The expression 2 denotes the output of hidden layer nodes. The formula 3 shows the final output.

$$
\begin{aligned}
& S_{j}=\sum_{i=1}^{m} w_{i j} x_{i}-\theta_{j}(j=1,2, \ldots, p) \\
& b_{j}=1 /\left[1+\exp \left(-\sum_{i=1}^{m} w_{i j} x_{i}+\theta_{j}\right)\right](j=1,2, \ldots, p) \\
& x_{i+1}=1 /\left[1+\exp \left(-\sum_{j=1}^{p} v_{j} b_{j}+\gamma\right)\right]
\end{aligned}
$$


In terms of data-based methods, incremental learning neural network models offer a number of benefits owing to their robust-ness in handling large scale data sets and their distributed learning capabilities. BP neural network in training before the connection weights and threshold of each layer randomly initialized value between $[0,1]$ which means this approach is without optimization random initialization and often can make the slow convergence speed of BP neural network and easy to make the final result for the optimal solution. Using the genetic algorithm can optimize the initial weights and threshold value distribution to optimize the initial weights and threshold can make the BP neural network has higher precision. The combination of the technique will be shown in the next section.

The Combination with Power System. Generator in phase adjustment process is a typical nonlinear process of power grid voltage. Generator leading phase operation and power grid is the most concerned in phase ability index when the angle of its voltage regulation effect whereas the size of the Angle is not only in phase plan to develop the basis and premise. At the same time, it is the important indicators of generator in phase stability. As the sequence of data samples could affect the formation of hyper boxes in FMM-based networks, a total of three runs, each with a randomized sequence of data samples, were conducted to illustrate the effects of data sequences on hyper box structures. Under the actual operating conditions (generator output active power and reactive power), angle prediction and regulating effect is the important topic of generator leading phase operation control. In this section, we design the RBFNN choice of generator active power and reactive power for the input and the generator power angle and power grid voltage for output which is issued by analysis of active power and reactive power regulating effect of motor into phase angle and the generator leading phase operation. Hidden layer neurons number is according to the group number of input samples and convergence speed combined with the convergence error compromise choice which should be noted that many in the input vector instead of too much on the number of hidden layer units can make the network is too big which will lead to network availability. Due to the selected input dimension and order of magnitude difference between samples are based on large difference between the numerical. So we need to normalization processing samples respectively to speed up the network training speed and prevent neurons by absolute value is too large net input saturation. The formula 3 and 4 show the restrictive conditions.

$$
\begin{aligned}
& X_{n}=\frac{2\left(X-X_{\min }\right)}{X_{\text {max }}-X_{\text {min }}}-1 \\
& Y_{n}=\frac{2\left(Y-Y_{\text {min }}\right)}{Y_{\text {max }}-Y_{\text {min }}}-1
\end{aligned}
$$

RBFNN generated automatically by the training to determine the required number of hidden layer units, from one neuron to begin training, increase network automatic neurons by examining the output error, the training sample after each cycle, use of network to produce maximum error of the training samples as the weight vector to add a new hidden layer neurons, and then recalculate, and check the updated network error. Repeat this process until the error square number is less than the target error or neurons to set maximum. It could be observed that the hyper box structures differed in the early stages of learning (at15\% and 30\%). However, the hyper box structures started to indicate the underlying data distributions when the number of data samples learned by MFMM became large (at 50\%). Finally, MFMM was able to represent stably the underlying data distributions with four hyper boxes (at 100\%), in response to randomized sequences of data samples. Through using Newton-Ralph calculation method for power systems which contain wind farms power flow calculation and reactive power compensation capacity calculation accuracy with the wind of the bus voltage compensation can meet the requirements of interconnection and stable operation. But in the process of calculation, the amount of every change that goes on the power flow calculation and the large amount of calculation and calculation process is complex and time consuming. It is not conducive to real-time reactive power compensation of wind farms implementation. Therefore, this article puts forward the wind farm reactive power compensation based on RBF neural network optimization algorithm. Even when the sequence of data samples is randomized, MFMM manages to 
capture the underlying data distributions, and formulates the hype box structures with the appropriate centroids accordingly. Based on the centroid information, the scores can be calculated. The simulation and corresponding experimental analysis are conducted in the following section.

\section{Experiment and Simulation}

In the figure 2, we show the numerical and demonstrative result of the proposed system. By the experimental results, the number of hidden layer neurons within a certain range increases will get corresponding to the convergence of error decreases. But as the number of hidden layer neurons increased from 14 to 18, the generalization error increase instead. Through investigating its reason, we conclude that the main factor is to use a large number of neurons to the output layer weights matrix pathological happen which affects modeling effect. According to the rule of Moody, under the same learning accuracy, the less effective parameters of the neural network, the better the neural network generalization ability is. The simulation result shows the feasibility of the proposed methodology.

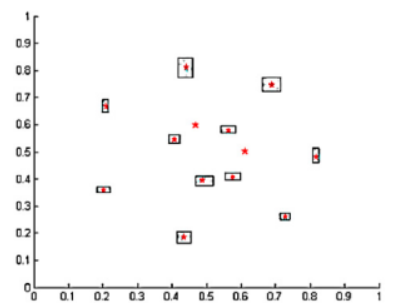

(a) Run $1,15 \%$

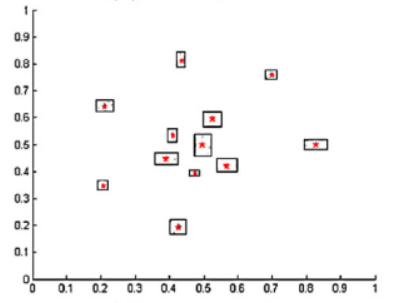

(a) Run $2,15 \%$

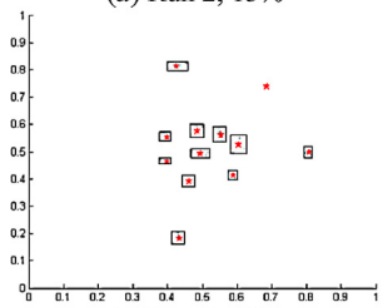

(a) Run $3,15 \%$

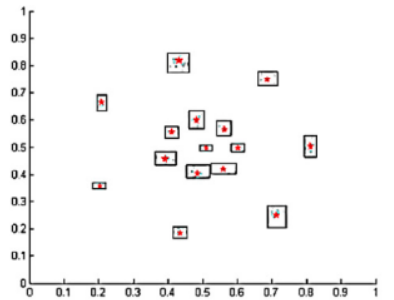

(b) Run $1,30 \%$

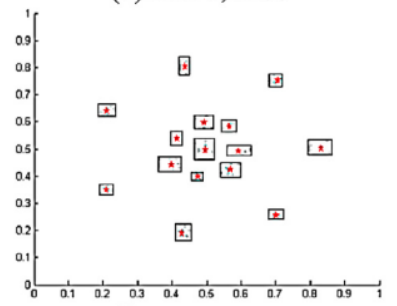

(b) Run $2,30 \%$

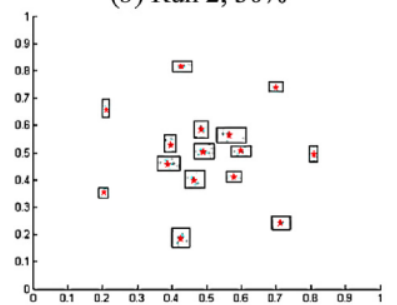

(b) Run $3,30 \%$

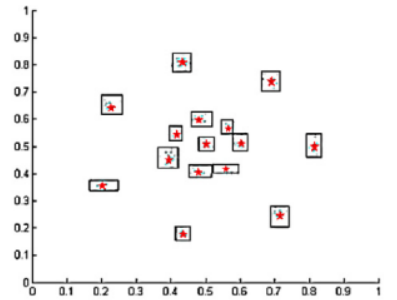

(c) Run $1,50 \%$

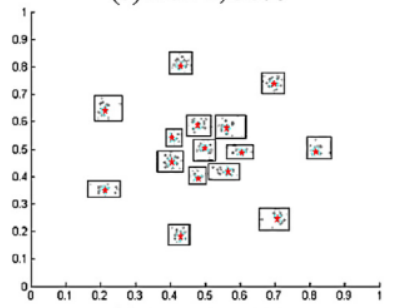

(c) Run $2,50 \%$

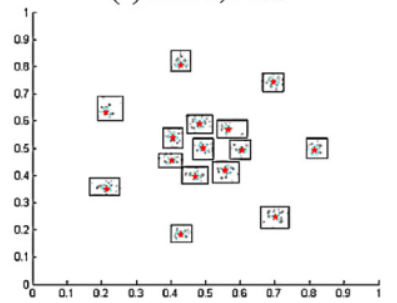

(c) Run $3,50 \%$

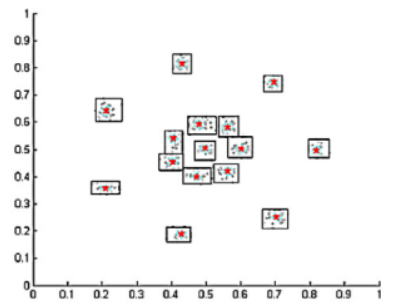

(d) Run $1,100 \%$

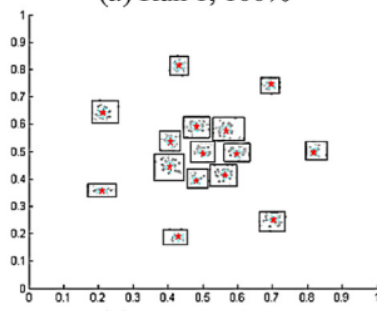

(d) Run $2,100 \%$

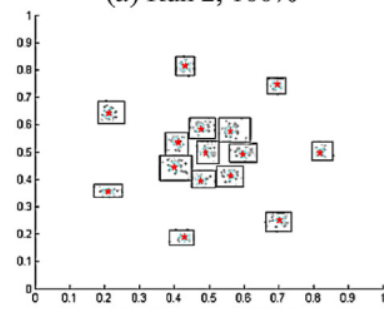

(d) Run 3, 100\%

Fig. 2 The Simulation Result for the Proposed Approach

\section{Conclusion and Summary}

Data analysis procedures can be broadly categorized as either exploratory or confirmatory, based on the models used for processing the data source. We argue that a filter bank that is designed from perceptual evidence is not always guaranteed to be the best filter bank in a statistical modeling framework where the end goal is word error rate. Therefore, to combine the proposed techniques with the neural network, we conduct theoretical analysis and numerical simulation on the power systems. Through theoretical analysis and numerical simulation, we prove the effectiveness of the proposed system with visual demonstration. In the future, we plan to conduct more related research to take advantage of the deep neural network and more complex power systems for the better result. 


\section{References}

[1] Hong, X., Chen, S., \& Harris, C. J. (2014). B-spline neural network based single-carrier frequency domain equalisation for Hammerstein channels. International Joint Conference on Neural Networks (pp.1834 - 1841). IEEE.

[2] Mikolov, T., Karafiát, M., Burget, L., Cernocký, J., \& Khudanpur, S. (2010, January). Recurrent neural network based language model. InINTERSPEECH 2010, 11th Annual Conference of the International Speech Communication Association, Makuhari, Chiba, Japan, September 26-30, 2010 (pp. 1045-1048).

[3] Zhang, H., Luo, Y., \& Liu, D. (2009). Neural-network-based near-optimal control for a class of discrete-time affine nonlinear systems with control constraints. Neural Networks, IEEE Transactions on, 20(9), 1490-1503.

[4] Tapkin, S. (2014). Estimation of fatigue lives of fly ash modified dense bituminous mixtures based on artificial neural networks. Mat Res, 17, 2, 316-325.

[5] Langer, N., Bhat, A. H., \& Agarwal, P. (2014). Neural-network-based space-vector pulse-width modulation for capacitor voltage balancing of threephase three-level improved power quality converter. Iet Power Electronics, 7, 4, 973 - 983.

[6] Nakisa, B., Nosratabadi, S. M., Rajabi, A., \& Dehghani, M. (2014). Synchronization of a microgrid with main network through static switch based on neural network controller. Conference on Electrical Power Distribution Networks (pp.94 - 99). IEEE.

[7] N S. A Novel Hybrid Classification Model of Genetic Algorithms, Modified k-Nearest Neighbor and Developed Backpropagation Neural Network.[J]. Plos One, 2014, 9. 